\title{
Automated Check-Post on Cloud using BigData Analysis with Web Service Security
}

\author{
Pankaj Kumar \\ Assistant Professor, \\ Dr. T. Thimmaiah Institute of \\ Technology, \\ Oorgaum, KGF-563120
}

\author{
Punitha F \\ Assistant Professor, \\ Dr. T. Thimmaiah Institute of \\ Technology, \\ Oorgaum, KGF-563120
}

\author{
Premlatha D \\ Assistant Professor, \\ Dr. T. Thimmaiah Institute of \\ Technology, \\ Oorgaum, KGF-563120
}

\begin{abstract}
In this Real world each and every check post perform the same operation. But the operation must be done manually. This operation includes various parameters. The manual operation of the check post requires the man power in order to perform the operations such as opening the gate, closing the gate, checking the vehicle details. The vehicle details include vehicle registration number, vehicle insurance, and vehicle fitness certification. It is a complex task to check each and every vehicle's details by a common man. Some people will do the task honestly and some may not. To overcome such complexities we can a build a system that performs these tasks on behalf of the man power. This system is known as Automated Check post system. The architecture of the system is service-oriented. This architecture will provide the several benefits in the real world. It is important to study the operations of all the devices that are required to build the system. After analyzing the operations then the next step is to check the performance of the system, taking into account the number of vehicles pass through the check post every day. We take a sample scenario on the automated check post system to check its feasibility solution and its performance. The collection of data will be so large and complex that companies cannot process it all using existing database systems, then BigData will play a key role in RFID's infrastructure for the industries needs for complete data management solutions with high security. The conclusion shows how much useful the system is, when it is implemented in the real word application.
\end{abstract}

\section{Keywords}

Automated check-post, RFID, Cloud computing, BigData analysis, GPS

\section{INTRODUCTION}

An automated check post is a system that has the unique technology of checking the details of vehicles. An automated check post system checks vehicle details like vehicle registration number, vehicle insurance, and vehicle fitness details. An automated system not only checks the vehicle details but also it provides authentication service. This system first checks the vehicle details and authenticates. If the details of the vehicle are valid then the check post gate will be unlocked by the system, suppose if the vehicles are not valid then the gate will not be unlocked. The activities of this system can be compared to the activities done by a common man operating the check post. The activities of this system are more efficient and effective when compared to the activities that are done manually. An automated check post system works based on the RFID (Radio Frequency Identification).

The RFIDs are also used for various different applications. In an automated check post system RFID plays an important role. The RFID has two main devices namely a RFID tag and a RFID reader. The main goal of the system is to collect the information about the vehicle and validate its information. This system will be a dummy system without a RFID.

In other words, RFID is an important parameter in this system.

\subsection{RFID}

RFID is a short form of "Radio Frequency Identification". RFID having two main functions namely reading the particular information and authenticating that information by comparing with the predefined factors. There are two main devices in the RFID system namely RFID tag and RFID reader. RFID tag is placed on the vehicle. RFID reader, which is present in the check post and is responsible for reading the information from a distance of certain meters. RFID is not same as the barcode. In the case of barcode the information can be read it and only if the barcode reader is placed very close to the barcode.

But in the case of RFID, the information can be read even if the RFID reader is placed certain meters away from the RFID tag. Hence RFID is not as same as a barcode. RFID tags transmit the identity to a small distance and they do not use any battery for transmitting the identity. On the other hand is the RFID reader which uses power for reading the identity from the RFID tag.

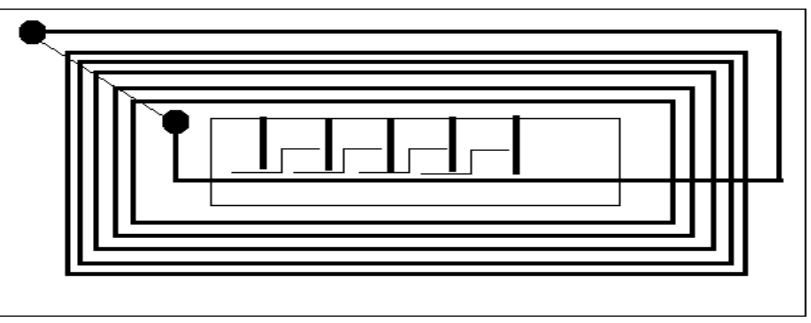

Figure 1: RFID tag

\section{Tng Render}

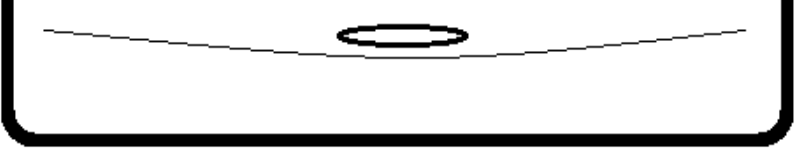

Figure 2: Tag Reader 
The above figure shows the tag reader. The tag reader receives the information from the tag reader when the tag is in the range of the tag reader.

\subsection{Wireless Sensor Network}

A Wireless Sensor Network consists of number of sensor nodes. These nodes can measure data, sense the data, and gather information from environment and those sends data to the destination sink. These sensor nodes continuously monitor a specific region to obtain data about the environment. In the dense deployment of wireless sensor networks there is innumerable traffic of packets which lead to congestion in the network. This nature of the sensor node is very dangerous because at a sudden event all the nodes detect and they become active and send the generated information to the destination sink. However the information which is transmitted has very high priority. This busy traffic that results from detected event can cause congestion because at that time all the nodes are active and try to send their generated data to destination sink. The sensor node sends two types of information 1.Periodic Data 2. Event Generated Data. Periodic Data is the data which sends data at equal interval of time, for example the data about the temperature of any city. Event generated data is the data which is send after generation of a specific events, for example the data of earth quake generation. When the congestion occurs in networks then the other quality of service parameters like throughput, reliability starts to decrease.

\subsection{BigData Challenged and need}

"Big data is collection of large data set and complex which is unable to process them using traditional data processing techniques.

The challenges include in traditional data processing is analysis, duration, capture, sharing, storage, search, visualization, transfer, and privacy violations. The larger data sets is due to the additional information derivable from analysis of a single large set of related data, as compared to separate smaller sets with the same total amount of data, allowing correlations to be found to "spot business trends, prevent diseases, combat crime and so on".

To improve these database system we need to use Hadoop cluster for search, Cloud computing as service oriented.

\section{RELATED WORKS}

The Various Designed system has given the solutions for transport and traffic control like traffic rule violation, parking system \& so on. It was proposed earlier low cost solution using RFID and GSM Technology. Later on RFID Technology consider using battery based and RFID tags to provide more robust and effective solutions. Now a day's the manual check post are the most commonly used in our country. There are many risks in the manual check post such as bribe, robbery, illegal transportation, movement of illegal vehicles, uninsured vehicles, and unregistered vehicles. To overcome with this problem we have proposed to use centralized server with high security and use the concept of BigData analysis due to data increases on server. All these scrap can be overcome by using An Automated Check Post Technique using BigData on cloud environment.

\section{PROPOSED ALGORITHM}

When proposing and developing an algorithm, shorter paths are preferred, as they usually means faster communication, but this approach usually cause to a negative performance in the Wireless sensor networks. If a shorter path is always selected, premature exhaustion of some nodes will occur, reducing the coverage area. Also, if a longer path is always chosen, the message that need to be transmitted would reach the sink node after a long delay and after travelling several intermediate nodes, something that cannot be allowed in mission-critical applications. Selecting the shortest path will frazzle prematurely the nodes, and selecting the longest will create undesirable long delays, so both extremities are not optimal and a middle solution is required for routing purposes. That's the main reason why pheromones are used to address the problem. In the algorithm, pheromone concentration measures the number of times traffic has passed through a node, and hence is used to calculate the energy content of that node, since the estimated remaining energy of a node is proportional to the inverse of pheromone concentration.

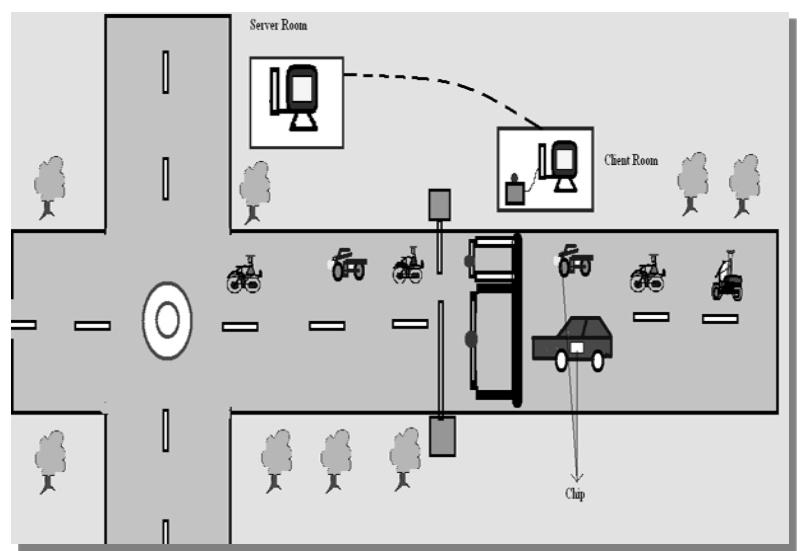

Figure 2. System Architecture of Automated Check Post

We need to add cloud services where the all node get connected. A handheld bar-code or RFID reader would be attached to dedicated enterprise applications, such as a warehouse management system (WMS) or a transportation management system (TMS) with defined business processes.

The Auto-ID of RFID, companies utilizing GS1 structured identifiers for product labelling to relate data captured when a customer scans a bar code or RFID Chip using a smartphone with legacy applications.

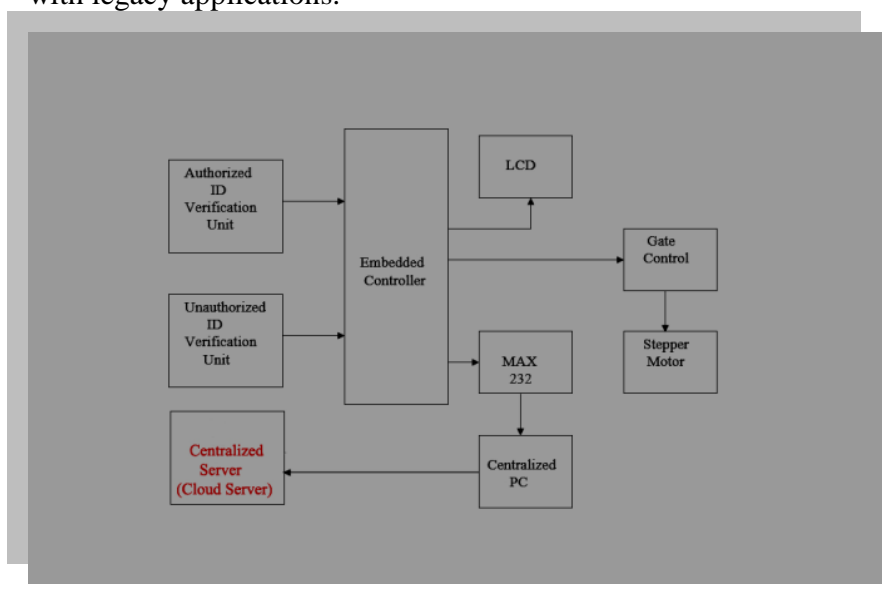

Figure 2.1. Architecture Diagram

By creating a set of APIs to store unique bar code or RFID chip using independent software, then all these data will be stored nearby could server which is centralized system. A Cloud computing is important approach, because neither the Internet of Things nor conventional machine-to-machine (M2M) approaches will likely be able scale across multiple cloud servers to represent networked systems with any 
communality and faster accessibility. By building models of legacy systems infrastructure, with APIs that can be accessed on the cloud, we can ease the task of moving information up and down. This is to be more scalable and efficient than pointto-point linkages between intermittently connected systems. Businesses prototyping such models-including smarttransportation, smart-grid and smart-manufacturing networks.

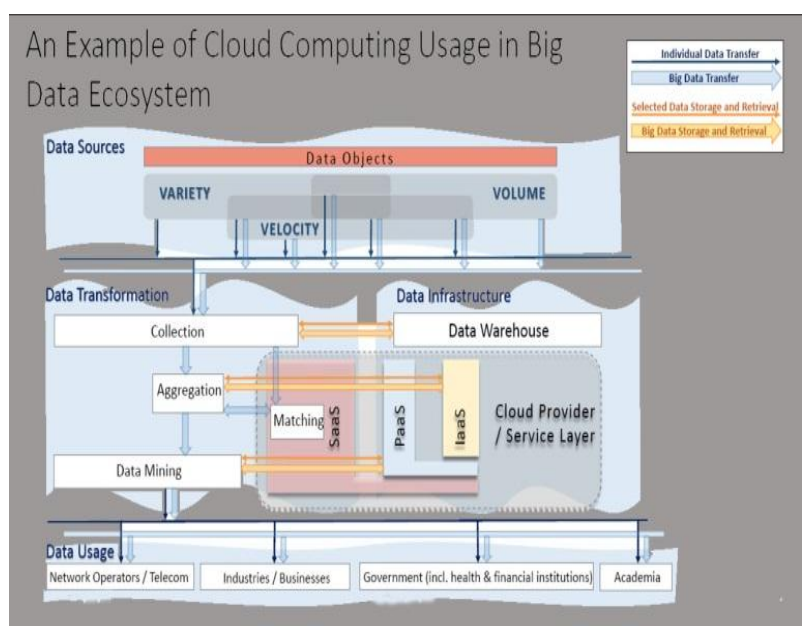

Fig3. Cloud computing using big data ecosystem

\subsection{Security Issue and Guideline}

In this module we have be careful from several attack on web server. Security pros draw a line at the firewall-what happens "out there" might be beyond their control, but a secure method is intended to protect the data and systems within. However, fails to take into account the role of developers, users and others along with the cloud computing systems, hardware, and software coming into the enterprise. It's called the cyber security assembly and acquisition, data sharing among server, governance, and more, to the security of IT systems and software. Here we are going to consider different types of attack.

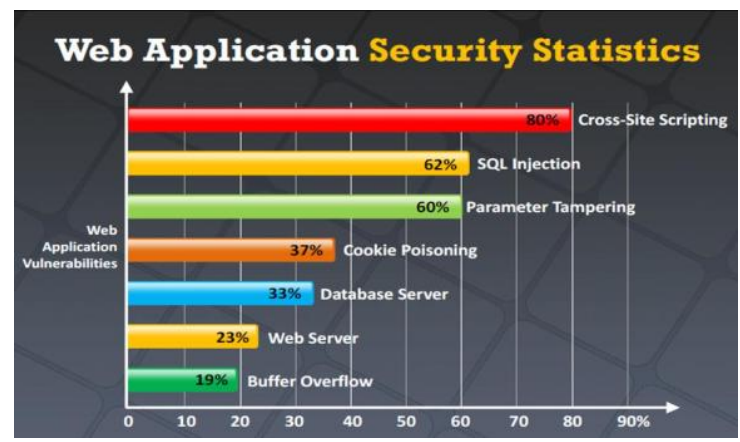

Figure 4. Web Security attack

\subsubsection{The Disabling Attack:}

In a disabling attack, the attacker causes to assume a state from which they can no longer be associate by the server. To avoid this is by having each tag stored in the server, a permanent (non-editable) private key. Then, the private key is read by a reader, it will generate a response using private key similar to match which is stored in server, it should be hard for an attacker to extract the private key from the tag's response. We need to use, a cryptographic system one-way function. This solution relies heavily on the server is trusted and physically secured.

\subsubsection{Cloning attack:}

To prevent these attacks it is not be possible for attackers to access identifying data. However for authentication, the backend server to verify a response and this must verify to identifying data (stored).

\subsubsection{The Tracking attack}

Unauthorized tracking is based on tracing to a particular tag. This can be avoid by that the values of the responses appear to an attacker as random. In fact, since we are assuming that all entities of an RFID system have polynomial bounded resources with values to be pseudo-random.

\subsubsection{Replay attacks}

To prevent replay attacks the response from RFID must be unique for every server. These values of the server responses must be unpredictable. We need to use pseudo-random.

\subsection{Security Guidelines:}

We assume that all authorized RFID readers are connected to a back-end server using a secure communication channel (authenticated and reliable). Each has two values: a private, key ktag, which it shares with the back-end server and a volatile identifying pseudonym rtag which is updated each time the tag is challenged. The server has a database $D$ in which it stores for each tag the pair of values (rtag, ktag) indexed by rtag - see the below figure Entity authentication protocol system.

$$
\begin{aligned}
& \operatorname{SeRver}(D) \leftrightarrow \operatorname{READER} \quad \operatorname{TAG}\left(k_{\text {tag }}, r_{\text {lag }}\right) \\
& r_{s y s} \\
& v \leftarrow F\left(k_{\text {lag }}, r_{\text {tag }} \| r_{\text {sys }}\right) \\
& \left(v_{1}, v_{2}\right) \stackrel{\text { parse }}{\longleftarrow} v \text {; } \\
& \bar{r}_{\text {tag }} \| r_{2} \quad\left(\bar{r}_{\text {tag }}, r_{\text {tag }}\right)-\left(r_{\text {tag }}, v_{1}\right) \\
& \text { if }\left(\bar{r}_{\text {tag }}, k_{i}\right) \in D \text { then range } \leftarrow[i: i] \\
& \text { else range }-[1: n] \\
& \text { for } j \text { in range do } \\
& v^{*}-F\left(k_{j}, \bar{r}_{t a g} \| r_{s y s}\right):\left(v_{1}^{*}, v_{2}^{*}\right) \stackrel{\text { parsan }}{\longleftarrow} v^{*} \\
& \text { if } v_{2}=v_{2}^{*} \text { then output ACCEPT }\left(\operatorname{tag}_{j}\right) \\
& \text { update } D:\left(r_{j}, k_{j}\right)-\left(v_{1}^{*}, k_{j}\right)
\end{aligned}
$$

Figure 5.Entity authentication protocol system

\subsection{Monitoring System}

In this monitoring system the back-end service provider can monitor the performance of their services using web based application or Smartphone. If a state change to object on human manipulation then it will be captured using sensor enabling activity-support services and it will send to mail to admin at urgency level.

\section{TERSO SOLUTIONS: AN EARLY CLOUD ADOPTER}

Terso is a third party paid application of services-based model only when they needed. Terso has shared the resources to as open source environment on salesforce.com. The RFID tags has placed into hospital inventory management and by medical device manufacturers and laboratories to control and monitor high value products to analyze and capture data and send to a centralized server. Once the data captured from front end, then the back-end process utilizes a business approach to provide global visibility of inventory and each item transaction traceability will be easy to use on web-based user 
interface. The advantage to integrate this RFID data with existing systems allowed for automation of standard business processes which will saved time and money and human resource with accuracy.

\section{CONCLUSIONS}

RFID is a challenging task platform from an information assurance standpoint .In Normal check post the time consumed to check the vehicle details is more when compared to the automated check post. In Automated check post unauthorised vehicles can be tracked. A RFID identification that simultaneously achieves security against tracking, cloning, and disabling and BigData will be managed on cloud with high end security. Integration features based on highly advanced IC's and processor with growing technology the project will be implemented

\subsection{Advantages}

1. Avoids driving of illegal vehicle.

2. Helps to track robbed vehicle.

3. Provides easy way to check vehicle documents.

4. Centralize database is avail to verify from anywhere

\section{FUTURE SCOPE}

This project will be implemented on basis of image processing to capture each movement on the move of vehicles. In our project we have implemented for vehicle detection system using RFID, further we can implemented camera based technology which will record the all movement as move the vehicle and it scan the entire vehicle to detect internally \& it will help to detect in crime cases like terrorism $\&$ smuggling goods and also it reduce the check-post load.

\section{ACKNOWLEDGMENTS}

The authors would like to thank to all co-author for helpful discussions, fundamental contributor in several of our related works.

\section{REFERENCES}

[1] G. Ateniese, J. Camenisch, and B. de Medeiros, ntraceable RFID tags via insubvertible encryption, Proc. ACM Conf. on Computer and Communication Security (ACM CCS 2005), ACM Press, 2005, pp. 92-101.
[2] Lejla Batina, Joseph Lano, Nele Mentens, Siddika Berna Ors, Bart Preneel, and Ingrid Verbauwhede, Energy, performance, area versus security tradeoffs for stream ciphers, The State of the Art of Stream Ciphers, Workshop Record, ECRYPT, 2004.

[3] Mihir Bellare, Anand Desai, Eron Jokipii, and Phillip Rogaway, A concrete security treatment of symmetric encryption: Analysis of the DES modes of operation, Proceedings of 38th Annual Symposium on Foundations of Computer Science (FOCS 97), IEEE Press, 1997, pp. pp. 394-403.

[4] M. Burmester, T. van Le, and B. de Medeiros, Provably secure ubiquitous systems: Universally composable RFID authentication protocols, Proceedings of the $2^{\text {nd }}$ IEEE/CreateNet International Conference on Security and Privacy in Communication Networks (SECURECOMM 2006), IEEE Press, 2006.

[5] Mike Burmester and Breno de Medeiros: RFID Security: Attacks, Countermeasures and Challenges

[6] Wikipedia-https://en.wikipedia.org/wiki/Big_data

[7] Mr. Pankaj Kumar Developing a Conceptual Relationship between Web Service Supply Chain Entities Using Web Security- International Journal of Computational Engineering Research (IJCER)-May, 2013

[8] Sewon oh, joosang park, yongioon lee, "rfid-based middleware system for automatic identification", IEEE international conference on service operations and logistics, and information, 2005.

[9] Kin seongleong, munlengng, member, IEEE, alfio r. Grasso, peter h. Cole, "synchronization of rfidreadersfor dense rfid reader environments", international symposium on applications and the internet workshop (saintw'06), 2005

[10] Implementation of Embedded System Using RFID and Alcohol Sensor at the Toll Plaza International Journal of Innovative Research in Computer and Communication Engineering-Vol. 2, Issue 4, April 2014 